\title{
ON CONNECTED AND REGULAR POINT SETS*
}

BY R. L. WILDER

In a recent number of this Bulletin $\mathrm{G}_{\mathrm{G}} \mathrm{T}$. Whyburn showed that if $A$ and $B$ are any two points of a connected and regular point set $M$, and $K$ denotes the set of all points of $M$ which separate $\ddagger A$ and $B$ in $M$, then $K+A+B$ is a closed and bounded point set. In the present paper I shall show that this theorem is susceptible of quite simple proof, and admits of two obvious generalizations which hold in space of $n$ dimensions. The methods used in proving these generalizations are also employed to show that if $N$ is a closed and bounded point set which lies in a connected subset of an open subset, $F$, of a connected and regular point set, $M$, then $F$ contains a bounded, connected and regular set which contains $N$. These results are then applied to give certain theorems concerning continuous curves.

Use will be made of the notions region of $M$ and simple chain of regions as introduced in my paper, The non-existence of a certain type of regular point set, $\$$ as well as of Theorem 1 of that paper; these extend readily to $n$-dimensional space, if in the definition of region of $M$ "circle" is replaced by "sphere." Furthermore, since the Borel property is employed I make note of the following lemma, the proof of which should be quite obvious.

* Presented to the Society, April 7, 1928.

$\dagger$ G. T. Whyburn, Concerning connected and regular point sets, this Bulletin, vol. 33 (1927), pp. 685-689.

$\ddagger$ If $A, B$ and $X$ are points of a connected set $M$, then $X$ is said to separate $A$ and $B$ in $M$ if $M-X$ is the sum of two mutually separated point sets which contain $A$ and $B$ respectively.

$\S$ This Bulletin, vol. 33 (1927), pp. 439-446. This paper will be referred to hereafter as N. E. R.

\|I It will be understood hereafter without explicit statement that the results hold for sets imbedded in euclidean space of $n$ dimensions. 
LEMмA. If $M$ is a connected and regular point set, and $N$ is a closed and bounded subset of $M$, and $G$ is a collection of regions of $M$ which cover $N$, then $G$ contains a finite subset which covers $N$.

(By virtue of the property of regularity, there exists, concentric with the sphere used in determining a region $R$ of $M$, a sphere $S$ such that all points of $M$ interior to $S$ are also points of $R$. When it is said, then, that a collection of regions, $G$, covers a subset $N$ of $M$, it is meant that every point of $N$ is within the sphere $S$ which corresponds to some region of the collection $G$.)

Definition. If $N$ is a subset of a connected set $M$ and $P$ is a point of $M-N$, then $P$ is said to separate $N$ in $M$ if $M-P$ is the sum of two mutually separated sets each of which contains at least one point of $N$.

THEOREM 1. If $N$ is a closed and bounded subset of a connected and regular point set $M$ and $K$ denotes the set of all points which separate $N$ in $M$, then $K+N$ is a closed and bounded point set.

Proof. Suppose the set $K+N$ is not closed. Then there is a point $P$ which is a limit point of this set and which does not belong to it. As $N$ is closed, $P$ is a limit point of $K$, but not of $N$. If $x$ is any point of $M$, distinct from $P$ in case $P$ is a point of $M$, there exists a region of $M$ which covers $x$ and which neither contains $P$ nor has $P$ as a limit point. Let $G$ denote the collection of all such regions. By the above lemma, $G$ contains a finite set of regions, $R_{1}$, $R_{2}, \cdots, R_{n}$, such that every point of $N$ is in at least one of these regions. For every $i(i=1,2, \cdots, n)$, let $P_{i}$ be a point of $N$ in $R_{i}$. For every two points $P_{i}$ and $P_{i+1}(i=1,2, \cdots$, $n-1)$ there exists, by Theorem 1 of N. E. R., and by virtue of the fact that $P$ does not separate these points in $M{ }^{*}$ a

* Use is made in this connection and in the proof of Theorem 2 below, of Lemma 2 of my paper $A$ characteristization of continuous curves by a property of their open subsets, Fundamenta Mathematicae, vol. 11(1928), pp. 127-131. 
simple chain of regions of $G$ from $P_{i}$ to $P_{i+1}$. The regions $R_{1}, R_{2}, \ldots, R_{n}$ together with the regions which go to make up these simple chains form a finite set, $g$, of regions of $G$, and if $H$ denotes the set of all points of $M$ contained in regions of $g$, then $H$ is a connected and bounded set. As $P$ is not a limit point of $H$ there exist in the vicinity of $P$ points of $K$ which are not in $H$. But clearly such points cannot separate $N$ in $M$ since $N$ is a subset of $H$. Thus the supposition that $K+N$ is not closed leads to a contradiction.

To show that $K+N$ is bounded, let $G$ be any collection of regions of $M$ covering $N$ and proceed as above to establish the existence of a bounded connected set, $H$, which is a subset of $M$ and contains $N$. As every point of $K$ must lie in $H$, it is clear that $K+N$ is bounded.

Definition. If $A$ and $B$ are any two distinct subsets of a connected set $M$, and $X$ is a point of $M-(A+B)$, then $X$ is said to separate $A$ from $B$ in $M$ if $M-X$ is the sum of two mutually separated sets which contain $A$ and $B$, respectively.

Theorem 2. If $A$ and $B$ are any two distinct subsets of $a$ connected and regular point set $M$, such that $A+B$ is closed and bounded, and $K$ denotes the set of all points which separate $A$ from $B$ in $M$, then $K+A+B$ is a closed and bounded set.

Indication of Proof. Select $P$ and $G$ as in Theorem 1 . As $P$ does not separate $A$ from $B$ in $M$, there is a connected subset of $M-P$ which contains a point $P_{1}$ of $A$ and a point $P_{2}$ of $B$. Hence from $G$ can be selected a simple chain from $P_{1}$ to $P_{2}$.

The corollarys of Theorems 1 and 2 applied to continuous curves* are obvious.

As a direct consequence of Theorems 1 and 2 and of the fact that the difference of two closed sets is both an $F_{\sigma}$ and a $G_{\delta}, \dagger$ we have the following theorem.

${ }^{*}$ In this paper a continuous curve is considered as a closed, connected and regular point set not necessarily bounded.

$\dagger$ See F. Hausdorff, Grundzilge der Mengenlehre, Leipzig, 1914, p. 306. An $F_{\sigma}$ is the sum of a denumerable set of closed sets and a $G_{\delta}$ is the set of points common to a denumerable set of open sets. 
THEOREM 3. Let $M$ be a connected and regular point set. Then if $N$ is any closed and bounded subset of $M$, the set of all points which separate $N$ in $M$ is both an $F_{\sigma}$ and $a G_{\delta}$ : and if $A$ and $B$ are any two distinct subsets of $M$ such that $A+B$ is closed and bounded, the set of all points which separate $A$ from $B$ in $M$ is both an $F_{\sigma}$ and $a G_{\delta}$.*

By methods similar to those used in proving Theorem 1 and noting in addition the fact that a region in a connected and regular point set is itself a regular set (Theorem 2 of N. E. R.), we have the following result.

THEOREM 4. If $K$ is a closed subset of a connected and regular point set $M$ and $N$ is a closed and bounded subset of a connected subset of $M-K$, then $N$ lies in a bounded, connected and regular subset of $M-K$ which has no limit point in $K$.

The analog of Theorem 4 for continuous curves may be stated as follows.

THEOREM 5. If $N$ is a closed and bounded subset of an open subset, $Q$, of a continuous curve $M$, and $N$ lies in some connected subset of $Q$, then $N$ lies in a bounded continuous curve which is a subset of $Q$.

Proof. Hahn has shown $\dagger$ that if $P$ is any point of $M$ and $r$ is any positive number, there exists a continuous curve $M(P, r)$ which is a subset of $M$, contains every point of $M$ less than a certain distance $d$ (dependent on $r$ ) from $P$, and is such that all of its points are at a distance less than $r$ from $P$. The set of all points $\{x\}$ of $M$ such that $x$ is joined to $P$ by a connected subset of $M$ every point of which is at a distance less than $d$ from $P$ constitutes a region of $M$ and

* In this connection it may be of interest to note that it has been shown that the set of all cut points of a continuous curve is an $F_{\sigma}$. See C. Zarankiewicz, Sur les points de division dans les ensembles connexes, Fundamenta Mathematicae, vol. 9 (1927), pp. 124-171, Theorem 17.

$\dagger \mathrm{H}$. Hahn, Mengentheoretische Charakterisierung der stetigen Kurve, Wiener Akademie Sitzungsberichte, vol. 123, Part IIa, pp. 2433-2489; see Theorem XXI, p. 2475. Although Hahn states his result for a bounded regular continuum, it is clear that it holds for any regular continuum. 
will be denoted by $M^{\prime}(P, r)$. Clearly $M^{\prime}(P, r)$ is a subset of $M(P, r)$.

If $P$ is any point of $Q$, let $r$ be a positive number less than the distance* from $P$ to $M-Q$. Then the sets $M(P, r)$ and $M^{\prime}(P, r)$ are subsets of $Q$. By a method similar to that used in proving Theorem 1 it can be shown that there exists a connected set $H^{\prime}$ which consists of a finite number of the regions of type $M^{\prime}(P, r)$ and contains $N$. The set $H$ composed of the sets $M(P, r)$ associated with those sets $M^{\prime}(P, r)$ which constitute $H^{\prime}$ is a bounded continuous curve lying in $Q$ and containing all points of $N$.

Theorem 5 is a generalization of a result obtained by $R$. L. Moore to the effect that if $Q$ is an open subset of a continuous curve and $A$ and $B$ are two points which lie in a connected subset of $Q$, then $A$ and $B$ are joined by a simple continuous arc which lies wholly in $Q$. As I have shown elsewhere that this property is sufficient that a continuum be a continuous curve, it follows that the property stated in Theorem 5 also serves to characterize a continuous curve.

As I have indicated in a recent paper, $\S$ subsets of a point set $M$ may be separated in $M$ in different senses. For our present purposes we employ the following definitions.

Definition. If $N$ is a subset of a connected set $M$ and $P$ is a point of $M-N$, then $P$ is said to separate $N$ in $M$ in the weak sense if there exist two points of $N$ which do not lie in a connected subset of $M-P$.

* That is, the greatest lower bound of all distances $P x$, where $x$ is a point of $M-Q$.

$\dagger$ Concerning continuous curves in the plane, Mathematische Zeitschrift, vol. 15 (1922), pp. 254-260, Theorem 1.

$\ddagger$ Concerning continuous curves, Fundamenta Mathematicae, vol. 7 (1925), pp. 340-377, Theorem 18.

$\S A$ characterization of continuous curves by a property of their open subsets, Fundamenta Mathematicae, vol. 11 (1928), pp. 127-131. The terminology "separates in the strong (or weak) sense" should not be confused with "disconnects in the strong (or weak) sense" as introduced by R. L. Moore in Concerning the cut-points of continuous curves and of other closed and connected point-sets, Proceedings of the National Academy of Sciences, vol. 9 (1923), pp. 101-106. 
Definition. If $A$ and $B$ are any two distinct subsets of a connected set $M$ and $X$ is a point of $M-(A+B)$, then $X$ is said to separate $A$ from $B$ in $M$ in the weak sense if $M-Y$ contains no connected subset which contains points of betn $A$ and $B$.

As examples of cases where these two definitions of "separate" are satisfied, but where the preceding definitions* are not satisfied, consider the following examples.

Examples. Let $M_{n}(n=1,2,3, \cdots)$ denote the straight line interval joining the points $(0,0)$ and $(1,1 / n)$. Also, denote the points $(1 / 2,0),(1,0)$ and $(0,0)$ by $A, B$, and $X$, respectively, and let $M=A+B+\sum_{n=1}^{\infty} M_{n}$.

The set $M$ is connected, but the set $M-X$ contains no connected set containing $A$ and $B$. Hence $X$ separates $A$ from $B$ in the weak sense. However, there is no separation of $M-X$ into two mutually separated subsets containing $A$ and $B$, respectively, and thus $X$ does not separate $A$ from $B$ in $M$ in the strong sense, that is, in the sense of the definition which immediately precedes Theorem 2 .

If we let $N$ denote the set of all points with rational coordinates in the interval $[0,1]$ of the $X$ axis, except $(0,0)$, and define $M_{n}$ and $X$ as above, and let $M$ now denote the set $N+\sum_{n=1}^{\infty} M_{n}$, it is easy to see that $X$ separates $N$ in $M$ in the weak sense, but not in the strong sense, i.e., in the sense of the definition preceding Theorem 1 .

On the basis of Lemma 2 of my paper $A$ characterization of continuous curves by a property of their open subsets $\dagger$ we have the following extension of Theorems 1-5.

THEOREM 6. (1) Theorems 1, 2 and 3 still hold true if "separate" be interpreted to mean "separate in the weak sense"; (2) Theorem 4 still holds true if the words " $N$ is a closed and bounded subset of a connected subset of $M-K$ " be replaced by " $N$ is a closed and bounded subset of $M-K$ such that there is no separation of $M-K$ into two mutually separated sets each

* That is, the definitions preceding Theorems 1 and 2, respectively.

$\dagger$ Loc. cit. 
of which contains points of $N$;" and (3) Theorem 5 still holds true if the words " $N$ lies in some connected subset of $Q$ " be replaced by "there is no separation of $Q$ into two mutually separated sets each of which contains points of $N . "$

In conclusion we may note the following application of part (1) of Theorem 6 to the theory of irreducibly connected sets.*

THEOREM 7. If the connected and regular point set $M$ is irreducibly connected about a closed and bounded set $N$, then $M$ is a bounded continuous curve.

Proof. Let $P$ be any point of $M-N$. Then $M-P$ contains no connected subset which contains $N$, since $M$ is irreducibly connected about $N$. That is, $M-N$ is the set of points of $M$ which separate $N$ in $M$ in the weak sense, and accordingly by Theorem 6 , part (1), the set $(M-N)+N=M$ is bounded and closed. Hence $M$ is a bounded continuous curve.

It may be pointed out that Whyburn's Theorem $2 \nmid$ to the effect that if a connected and regular point set $M$ is irreducibly connected between two of its points $A$ and $B$, then $M$ is a simple continuous arc from $A$ to $B$, is a corollary of Theorem 7 above. For since by Theorem 7 such a set, $M$, is a continuous curve, $A$ and $B$ are the end points of a simple continuous arc, $t$, of $M$. It is clear, then, that $M \equiv t$.

The University of Mrchigan

* A connected set $M$ is said to be irreducibly connected about one of its subsets, $N$, if it has no proper connected subset which contains $N$. See H. M. Gehman, Concerning irreducibly connected sets and irreducible continua, Proceedings of the National Academy of Sciences, vol. 12 (1926), pp. 544-547.

$\dagger$ Loc. cit. I might say here that in establishing the first of those results concerning simple closed curves to which Professor Whyburn kindly calls attention in this connection, I found it necessary to prove as a lemma the definition of arc stated in his Theorem 2. I did not mention this in my abstract (this Bulletin, vol. 32 (1926), p. 123, abstract No. 15) and have not yet published the paper. However, the proof which I developed in that connection is quite different from that given by Professor Whyburn as well as from the proof indicated in the present paper. 